\title{
Multi-Objective Genetic Algorithm Optimization for Image Watermarking Based on Singular Value Decomposition and Lifting Wavelet Transform
}

\author{
Khaled Loukhaoukha, Jean-Yves Chouinard, and Mohamed Haj Taieb \\ Laval University, Quebec, QC, Canada, G1K 7P4 \\ khaled.loukhaoukha.1@ulaval.ca, jean-yves.chouinard@gel.ulaval.ca, \\ mohamed.haj-taieb.1@ulaval.ca
}

\begin{abstract}
In this paper, a new optimal watermarking scheme based on singular value decomposition (SVD) and lifting wavelet transform (LWT) using multi-objective genetic algorithm optimization (MOGAO) is presented. The singular values of the watermark is embedded in a detail subband of host image. To achieve the highest possible robustness without losing watermark transparency, multiple scaling factors (MSF) are used instead of single scaling factor (SSF). Determining the optimal values of the MSFs is a difficult problem. However, to find this values a multi-objective genetic algorithm optimization is used. Experimental results show a much improved performance in term of transparency and robustness of the proposed method compared to others methods.
\end{abstract}

Keywords: Digital watermarking, multi-objective optimization, genetic algorithm, singular value decomposition, lifting wavelet transform.

\section{Introduction}

In the digital era, it has become easier to exchange illegally digital multimedia content. In this context, digital watermarking was introduced as technical security protection solution which consists of inscribing invisible secret information (known as watermark) into multimedia content. In the case of digital images, watermarks are generally embedded in spatial or frequency domain. The spatial domain has advantage of low calculation complexity compared to transform domains. However, it suffers from a weak robustness against various attacks. Embedding watermarks in the frequency domain enhances the imperceptibility because the Human Visual System (HVS) behavior naturally follows the spectral characteristics (frequency domain) of the source. In recent years, artificial intelligence techniques has been used to improve the performances of digital watermarking methods [1 3]. This paper investigates genetic algorithm as artificial intelligence technique. The paper is organized as follow. In section 2, fundamental concepts of a genetic algorithm are explained. Section 3 describes

A. Elmoataz et al. (Eds.): ICISP 2010, LNCS 6134, pp. $394-403,2010$.
(c) Springer-Verlag Berlin Heidelberg 2010 
the SVD-LWT watermarking algorithm. In section 4, we explain how the multiobjective genetic algorithm optimization is used in the watermarking algorithm. The experimental results are discussed in section 5 and concluding remarks are given in section 6

\section{Fundamental Concepts of Genetic Algorithm}

Holland [4] defined the genetic algorithm (GA) as a technique based on the process of natural selection and genetic recombination and evolution. The main techniques employed in genetic algorithm are :

1. Population initialization : an initial population is a set of chromosomes which represents a solution of the problem. This population can be generated randomly or by some problem-specific heuristic. It important to note that the chromosome is expressed as a string of genes, which can be represented, depending on the application, by the binary alphabet, integers or real numbers.

2. Evaluation : this consists of classifying the chromosomes from best to worst according their fitness values, in order to reflects their importance in forming the next generation. This value is computed using an objective function.

3. Reproduction : this allows a chromosome the possibility to form the next generation. The probability that a chromosome will be chosen is based on its fitness value. This operator plays an important role in driving the search towards a better solution and maintaining diversity in the population. The main reproduction methods used are : roulette wheel selection and tournament selection.

4. Crossover : this combines pairs of chromosomes (called parents) to generate new chromosomes pairs (called offsprings) that share some features taken from the parents. The crossover is executed at fairly high probability (i.e. $\left.P_{C}>0.7\right)$. The aim of the crossover is to form a new generation with a higher proportion of the characteristics of the good chromosomes than the previous generation. There are many ways of doing the crossover, such as multi-points crossover, uniform crossover, and arithmetic crossover.

5. Mutation : this applies to some offspring chromosomes. It consists of introducing variations in the values of several genes selected at random. This operator ensures genetic diversity within the population and it is performed with a low probability (i.e. $P_{M}$ is kept within the range 0.001-0.05).

A classical genetic algorithm scheme is shown in figure 1

\section{Watermarking Algorithm Based on SVD and LWT}

The developed SVD-LWT watermarking algorithm [7] can be described by two processes : watermark embedding and watermark extraction. 


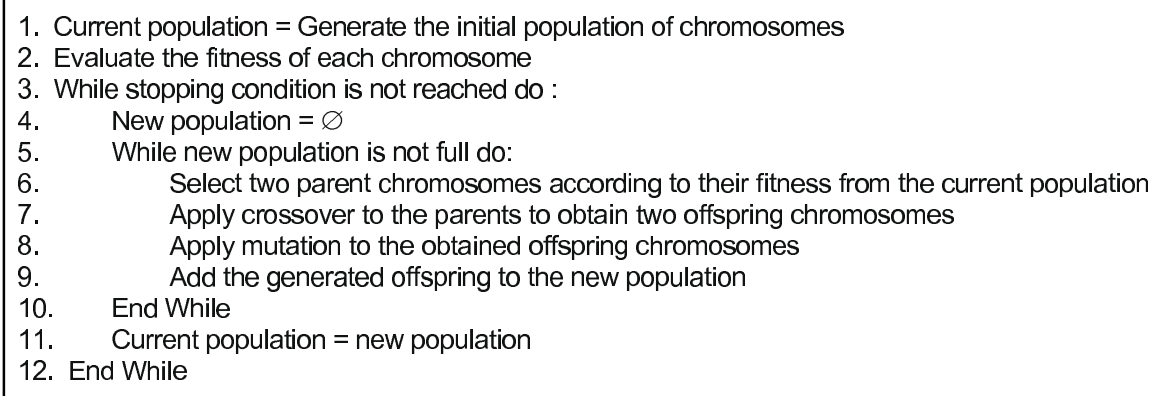

Fig. 1. Scheme of a classical genetic algorithm [6]

\subsection{Watermark Embedding}

Consider an original image $X$ of size $N \times N$ and let the watermark $W$ be a binary image of size $M \times M$. The embedding procedure is described as follows:

1. Decompose the original image $I$ into $3 \ell+1$ subbands by applying a $\ell$-levels lifting wavelet transform (LWT).

2. Select one subband $(S B)$ among the three following subbands: $H H_{\ell}, H L_{\ell}$ and $L H_{\ell}$.

3. Compute the inverse LWT of the selected subband $(S B)$ :

$$
X=L W T^{-1}(S B)
$$

4. Apply a singular value decomposition (SVD) of matrix $X$ :

$$
X=U_{X} \cdot S_{X} \cdot V_{X}^{T}
$$

5. Apply a singular value decomposition of the watermark matrix $W$.

$$
W=U_{W} \cdot S_{W} \cdot V_{W}^{T}
$$

6. Compute the one-way hash function to matrices $U_{W}$ and $U_{W}$ :

$$
\left\{\begin{array}{l}
H_{U}=\operatorname{Hash}\left(U_{W}\right) \\
H_{V}=\operatorname{Hash}\left(V_{W}\right)
\end{array}\right.
$$

7. The matrices $U_{W}$ and $U_{W}$, and the hash values $H_{U}$ and $H_{V}$ are stored in the private key.

8. Compute matrix $S_{Y}$ according to:

$$
S_{Y}(i, j)=S_{X}(i, j)+\alpha \cdot S_{W}(i, j), \quad 0 \leq i, j \leq M,
$$

Where $\alpha$ is the watermark strength factor that controls the tradeoff between visual quality and robustness of the watermarking scheme. 
9. Compute matrix $Z_{W}$, according to:

$$
Z_{W}=U_{X} \cdot S_{Y} \cdot V_{X}^{T}
$$

10. Compute the lifting wavelet transform of the matrix $Z_{W}$,

$$
S B_{W}=L W T\left(Z_{W}\right)
$$

11. The watermarked image $I_{W}$ is computed by applying the inverse lifting wavelet transform into the $\ell$-levels, to the modified subband $S B_{W}$ and the $3 \ell$ unmodified subbands.

\subsection{Watermark Extraction}

Several watermarking algoritm based on the singular value decomposition (SVD) which embed a watermark using singular values suffers from the high probability of false positive detections. This vulnerability in watermarking algorithm based on SVD was reported by Zhang et al. [8] and Rykaczewski [9] for the SVDbased spatial watermarking algorithm proposed by Liu and Tan [10]. Moreover, other researchers have mentioned that others algorithms suffers from the high probability of false positive detection [11, 12]. Since the proposed watermarking algorithm is based on singular value decomposition (SVD) and to protect it against this vulnerability, we used a solution proposed in [13] which consist in using a one-way hash function or encryption function. Briefly the proposed solution consists in storing the hash values of the singular vectors matrices during the watermark embedding process, these values are denoted by $H_{U}$ and $H_{V}$. Before starting the watermark extraction, a safety test is done. The following steps summarizes the extracting algorithm:

1. Safety test : the hash values of the singular vectors matrices $U_{W}$ and $V_{W}$ (probably changed by attacker to be $\tilde{U}_{W}$ and $\tilde{V}_{W}$ ) are computed. These hash values are denoted by $H_{\tilde{U}}$ and $H_{\tilde{V}}$ and compare them to the hash values stored in the embedding procedure (step 6). Then,

$$
\left\{\begin{array}{lll}
\text { if } \quad H_{U}=H_{\tilde{U}} \text { and } H_{V}=H_{\tilde{V}} \longrightarrow & \text { go to step 2 } \\
\text { if } \quad H_{U} \neq H_{\tilde{U}} \text { or } \quad H_{V} \neq H_{\tilde{V}} \longrightarrow \quad \text { stop algorithm attacked }
\end{array}\right.
$$

2. Decompose the original image $I$ and the watermarked image $I_{W}$, by applying the $\ell$-levels lifting wavelet transform.

3. Select the same subband $(S B)$ used in step 2 of the watermark embedding procedure. Let $S B_{I}$ and $S B_{I_{W}}$ are, respectively, the subbands seleceted for the original and watermarked images.

4. Compute the inverse lifting wavelet transform of the selected subbands $\left(S B_{I}\right.$ and $\left.S B_{I_{W}}\right)$ :

$$
\left\{\begin{array}{l}
X=L W T^{-1}\left(S B_{I}\right) \\
X_{I_{W}}=L W T^{-1}\left(S B_{I_{W}}\right)
\end{array}\right.
$$


5. Apply the singular value decomposition to the matrices $X$ and $X_{I_{W}}$ :

$$
\left\{\begin{array}{l}
X=U_{X} \cdot S_{X} \cdot V_{X}^{T} \\
X_{I_{W}}=U_{X_{I_{W}}} \cdot S_{X_{I_{W}}} \cdot V_{X_{I_{W}}}^{T} .
\end{array}\right.
$$

6. Compute matrix $S_{\hat{W}}$ as follows:

$$
S_{\hat{W}}=\frac{S_{X_{I_{W}}}-S_{X}}{\alpha}
$$

7. Determine the estimated watermark, $\hat{W}$, by computing:

$$
\hat{W}=\tilde{U}_{W} \cdot S_{\hat{W}} \cdot \tilde{V}_{W}^{T}
$$

\section{Optimal Watermarking Algorithm Using Multi-Objective Genetic Algorithm Optimization}

Watermarking schemes are either based on an additive or a multiplicative rule. The embedding rule are usually of the form (13) below.

$$
\left\{\begin{array}{lll}
I_{W}=I+\alpha \cdot W & \longrightarrow & \text { additive rule } \\
I_{W}=I \cdot(1+\alpha \cdot W) & \longrightarrow & \text { multiplicative rule }
\end{array}\right.
$$

where $I_{W}$ is (transformed) watermarked image, $I$ is the (transformed) original image and $\alpha$ is used to control watermarking strength. From (13), one can see that the watermark $W$ is scaled by factor $\alpha$ in the embedding process. Cox et al. [14] suggest the use of multiple scaling factors (MSF) instead of one. They state that considering a single scaling factor (SSF) may not be applicable for altering all of the values of original image $I$. Therefore, it is necessary to use multiple scaling factors (MSF) instead single scaling factor. Determining the optimal values of these factors, in order to achieve the highest robustness and transparency under various attacks, is unfortunately a difficult problem. In this paper, we propose a multi-objective genetic algorithm optimization (MOGAO) scheme in order to find the optimal multiple scaling factors (MSF) of the watermarking algorithm presented above in section 3. The steps for applying MOGAO into SVD-LWT watermarking scheme are:

1. Define the value of population size (PS), the number of variables (NV), the probability of crossover $\left(P_{C}\right)$, the probability of mutation $\left(P_{M}\right)$, the objective function, and the generation number (GN) or any other stopping condition.

2. Generate randomly an initial population of potential solutions.

3. Using embedding process, produce the watermarking images using each chromosomes of population.

4. Compute the normalized correlation between original and watermarked images, $N C\left(I, I_{W}\right)$, (the number of watermarked images $I_{W}$ is equal to PS). 
5. Apply the selected attacks upon the watermarked images $I_{W}$ one by one (T attacked images will be generated for each watermarked image).

6. Using the extraction process previously described, extract the watermarks from the attacked watermarked images.

7. Compute $N C(W, \hat{W}),(\hat{W}$ being the extracted watermark).

8. Evaluate an objective function for every chromosome of the population.

9. Apply the selection process, crossover and mutation operations.

10. Repeat steps 3 to 10 until the generation number is reached or another stopping condition is met.

The objective function is defined depending on imperceptibility, $N C\left(I, I_{W}\right)$, and robustness $N C\left(W, \hat{W}_{i}\right)$ (normalized correlation between the original watermark $W$ and the extracted watermark $\hat{W}$ under attack $i$, such as $i=1,2, \ldots, T$.). Equation (14) gives the fitness value of chromosome $i$.

$$
\text { Fitness }_{i}=\left[\frac{1}{N C\left(I, I_{W}\right)}+\frac{1}{N C(W, \hat{W})}-\frac{1}{T} \sum_{i=1}^{T} N C\left(W, \hat{W}_{i}\right)\right]
$$

It is clear from (14) that the ideal fitness value is one.

\section{Experimental Results}

In this section, several experiments are performed using three $256 \times 256$ grayscale images and a $32 \times 32$ binary watermark as depicted in figure 2 To show the effectiveness and the impact of the multi-objective genetic algorithm optimization in the proposed scheme, denoted as MSF-Scheme, our results are compared to the pure DWT watermarking scheme presented by [15] and the SVD-LWT watermarking scheme [7] as presented in section 3 but using a single scaling factor $\alpha$. These algorithms are denoted by PDWT and SSF, respectively. The embedding process is done in $\mathrm{LH}_{3}$ subband and the lifting wavelet transform levels $\ell$ is equal to 3 .

To determine the optimal values of multiple scaling factors (MSF), the genetic algorithm parameters should be selected carefully. These parameters (population size, crossover probability and mutation probability) are selected by varying one

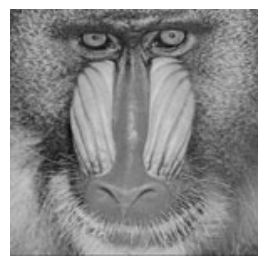

(a) Baboon

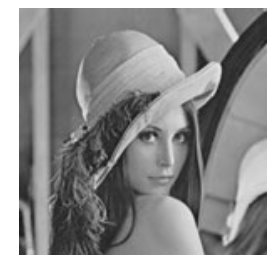

(b) Lena

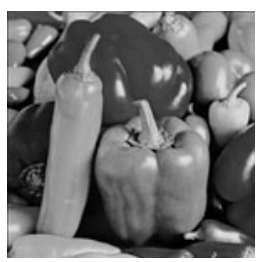

(c) Peppers

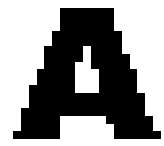

(d) Letter A

Fig. 2. Original (Baboon, Lena and Peppers) and watermark (Letter A) images 
Table 1. Parameters setting of the genetic algorithm

\begin{tabular}{|l|l|}
\hline Control parameters & Setting \\
\hline Population size (PS) & 100 \\
Generation number $(\mathrm{GN})$ & 150 \\
Number of variables $(\mathrm{NV}=\mathrm{MSF})$ & 32 \\
Selection method & Roulette Wheel selection \\
Crossover type & Arithmetic crossover \\
Crossover probability $\left(P_{C}\right)$ & 0.8 \\
Mutation type & Gaussian mutation \\
Mutation probability $\left(P_{M}\right)$ & 0.05 \\
\hline
\end{tabular}

parameter at a time while keeping the others fixed. The genetic algorithm setting parameters selected for simulation are listed in table 1.

For the experimental tests, eight different attacks are selected in conjunction to multi-objective optimization (i.e. $T=8$ ). These attacks are : salt \& peppers noise (density 0.05), Gaussian filter $(3 \times 3)$, cropping $(1 / 8$ center), JPEG compression $(Q=5)$, sharpening, scaling $(256 \rightarrow 512 \rightarrow 256)$, histogram equalization and gray value quantization (1 bit), denoted respectively by $\mathbf{S P}, \mathbf{G F}$, CR, CM, SH, SC, HE and QN. The proposed watermarking scheme is flexible: the number of selected attacks $(T)$ can be easily decreased or increased since the level of robustness differs according to the nature of the watermarking application. Table 2 gives the results of imperceptibility and robustness tests of the proposed scheme compared with the schemes proposed by Xianghong et al. [15] and Loukhaoukha et al. 7].

Table 2. Imperceptibility and robustness tests

\begin{tabular}{|c|c|c|c|c|c|c|c|c|c|c|c|}
\hline & \multirow{2}{*}{ Scheme } & \multirow{2}{*}{$\mathbf{N C}\left(\mathbf{I}, \mathbf{I}_{\mathbf{W}}\right)$} & \multirow{2}{*}{$\mathbf{N C}(\mathbf{W}, \hat{\mathbf{W}})$} & \multicolumn{8}{|c|}{$\mathbf{N C}\left(\mathbf{W}, \hat{\mathbf{W}}_{\mathbf{i}}\right)$} \\
\hline & & & & $\overline{\text { SP }}$ & $\overline{\text { GF }}$ & $\overline{C R}$ & CM & \begin{tabular}{|l|} 
SH \\
\end{tabular} & SC & $\overline{\mathrm{HE}}$ & QN \\
\hline \multirow[b]{3}{*}{ ๓ } & MSF-Scheme & 1.000 & 1.000 & 0.982 & 0.999 & 0.995 & 0.978 & 0.983 & 1.00 & 0.985 & 0.982 \\
\hline & SSF [7] & & 1 & 841 & 0.786 & 0.986 & 0.886 & 0.909 & 0.923 & 0.962 & 0.960 \\
\hline & PDWT [15] & 0.999 & 0.999 & 0.694 & 0.858 & 0.983 & 0.633 & 0.712 & 0.986 & 0.440 & 0.569 \\
\hline \multirow{3}{*}{ 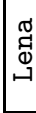 } & MSF-Scheme & 0.999 & 0.997 & 0.995 & 0.995 & 0.985 & 0.994 & 0.995 & 0.997 & 0.993 & 0.973 \\
\hline & $\mathrm{SSF}$ [7] & 0.999 & 1 & 0.749 & 0.703 & 0.835 & 0.856 & 0.964 & 0.993 & 0.986 & 0.969 \\
\hline & PDWT [15] & 0.999 & 0.999 & 0.616 & 0.866 & 0.983 & 0.640 & 0.666 & 0.994 & 0.587 & 0.625 \\
\hline \multirow{3}{*}{18} & MSF-Scheme & 0.999 & 1.000 & 0.985 & 0.997 & 0.977 & 0.946 & 0.987 & 1.000 & 0.989 & 0.978 \\
\hline & SSF [7] & 0.999 & 0.999 & 0.770 & 0.722 & 0.879 & 0.856 & 0.963 & 0.990 & 0.976 & 0.958 \\
\hline & PDWT [15] & 0.999 & 0.999 & 0.713 & 0.891 & 0.983 & 0.609 & 0.699 & 0.996 & 0.749 & 0.534 \\
\hline
\end{tabular}

From the table 2, one can see the impact of using MOGAO to find the optimal multiple scaling factors (MSF) in the proposed watermarking scheme compared to the same watermarking scheme using a single scaling factor (SSF). The experimental results presented above demonstrate clearly the much improved performance of the proposed watermarking scheme with multiple scaling factors 
Table 3. Optimal multiple scaling factors for Baboon image

\begin{tabular}{|c|c|c|c|c|c|c|c|c|c|c|c|c|c|c|c|c|}
\hline Rang & $\mathbf{1}$ & $\mathbf{2}$ & $\mathbf{3}$ & $\mathbf{4}$ & $\mathbf{5}$ & $\mathbf{6}$ & $\mathbf{7}$ & $\mathbf{8}$ & $\mathbf{9}$ & $\mathbf{1 0}$ & $\mathbf{1 1}$ & $\mathbf{1 2}$ & $\mathbf{1 3}$ & $\mathbf{1 4}$ & $\mathbf{1 5}$ & $\mathbf{1 6}$ \\
\hline MSF & 10.0 & 13.5 & 11.0 & 18.3 & 14.2 & 23.5 & 29.0 & -35.0 & -21.3 & -18.0 & -18.1 & 17.1 & -21.1 & -23.2 & -23.0 & 12.2 \\
\hline Rang & $\mathbf{1 7}$ & $\mathbf{1 8}$ & $\mathbf{1 9}$ & $\mathbf{2 0}$ & $\mathbf{2 1}$ & $\mathbf{2 2}$ & $\mathbf{2 3}$ & $\mathbf{2 4}$ & $\mathbf{2 5}$ & $\mathbf{2 6}$ & $\mathbf{2 7}$ & $\mathbf{2 8}$ & $\mathbf{2 9}$ & $\mathbf{3 0}$ & $\mathbf{3 1}$ & $\mathbf{3 2}$ \\
\hline MSF & 15.5 & -15.7 & -45.3 & 26.3 & 11.5 & -14.3 & 17.5 & -27.0 & -16.3 & 9.1 & 33.1 & -18.8 & -14.2 & 33.7 & -11.2 & -13.4 \\
\hline
\end{tabular}

(MSF) in terms of imperceptibility and robustness over the same watermarking algorithm using a single scaling factor (SSF) and Xianghong et al. [15] scheme.

Table 3 provides the optimal multiple scaling factors found by multi-objective genetic algorithm optimization (MOGAO) when the Baboon image is used as original image. Figure 3 depicts the watermarked images attacked and the extracted watermarks under a different attacks.
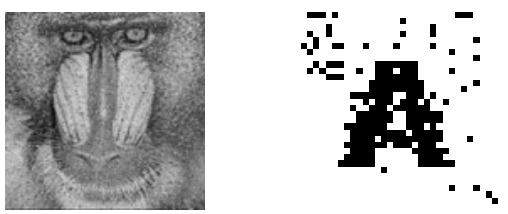

(a) Salt \& peppers noise $(\mathrm{NC}=0.951)$
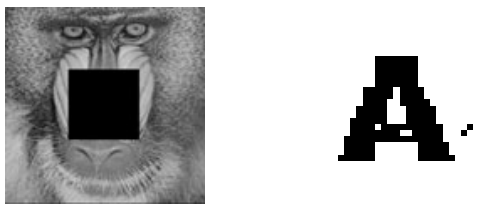

(c) Cropping $(\mathrm{NC}=0.994)$
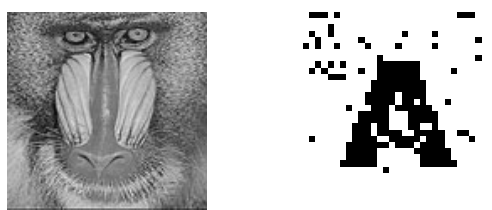

(e) Sharpening $(\mathrm{NC}=0.970)$
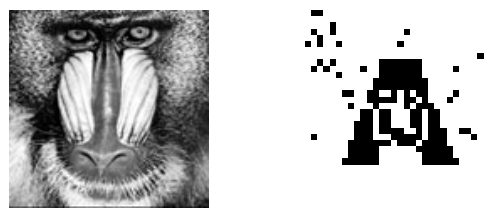

(g) Histogram equalization $(\mathrm{NC}=0.969)$
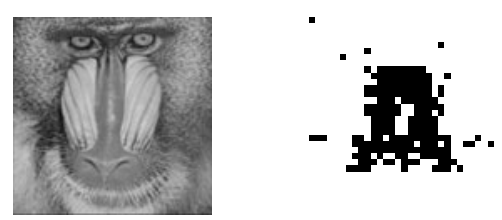

(b) Gaussian filtering $(\mathrm{NC}=0.963)$
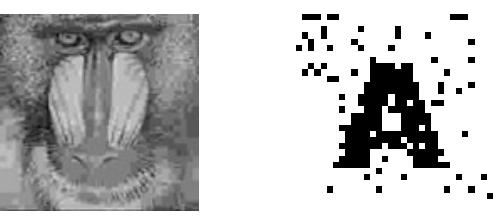

(d) JPEG Compression $(\mathrm{NC}=0.967)$
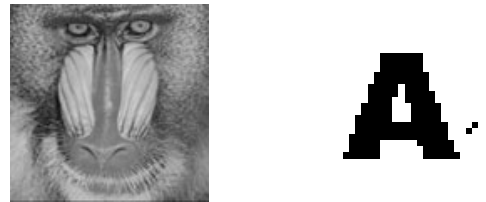

(f) Scaling $(\mathrm{NC}=0.998)$
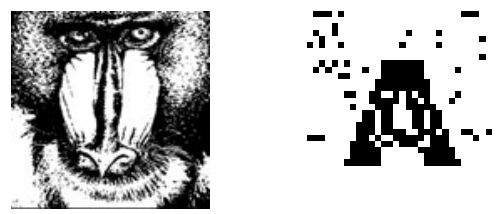

(h) Gray quantization $(\mathrm{NC}=0.966)$

Fig. 3. Watermarked image attacked under the 8 different attacks and corresponding extracted watermark 


\section{Conclusion}

Most watermarking schemes use additive or multiplicative embedding rules with a single factor $\alpha$ in order to get a tradeoff between robustness and imperceptibility. In this paper, a new optimal image watermarking scheme, based on SVD and LWT, using an additive embedding rule with multiple scaling factors (MSF) is proposed. To achieve the best trade-off in terms of robustness and imperceptibility, multi-objective genetic algorithm optimization is employed in order to determine the optimum values of MSF. Experimental results demonstrate that the proposed scheme outperforms the watermarking schemes proposed in [7, 15]. Moreover, the problem of false positive detection which affects most SVD-watermarking algorithms is solved using one-way hash functions.

\section{References}

1. Zhang, F., Zhang, H.: Applications of a neural network to watermarking capacity of digital image. Neurocomputing 67, 345-349 (2005)

2. Wang, Z., Sun, X., Zhang, D.: A novel watermarking scheme based on PSO algorithm. In: Proceedings of International Conference on Life System Modeling and Simulation, September 2007, pp. 307-314 (2007)

3. Kumsawat, P., Attakitmongcol, K., Srikaew, A.: A new approach for optimization in image watermarking by using genetic algorithms. IEEE Transactions on Signal Processing 53(12), 4707-4719 (2005)

4. Holland, J.H.: Adaptation in Natural and Artificial Systems. University of Michigan Press (1975)

5. Michalewicz, Z.: Genetic Algorithms + Data Structures = Evolution Programs.. Springer, Berlin (1996)

6. Garca-Villoria, A., Pastor, R.: Solving the response time variability problem by means of a genetic algorithm. European Journal of Operational Research 202(2), 320-327 (2010)

7. Loukhaoukha, K., Chouinard, J.Y.: Hybrid watermarking algorithm based on svd and lifting wavelet transform for ownership verification. In: Canadian Workshop on Information Theory, May 2009, pp. 177-182 (2009)

8. Zhang, X.P., Li, K.: Comments on an SVD-based watermarking scheme for protecting rightful ownership. IEEE Transactions on Multimedia 7(3), 593-594 (2005)

9. Rykaczewski, R.: Comments on an SVD-based watermarking scheme for protecting rightful ownership. IEEE Transactions on Multimedia 9(2), 421-423 (2007)

10. Liu, R., Tan, T.: A SVD-based watermarking scheme for protecting rightful ownership. IEEE Transactions on Multimedia 4(1), 121-128 (2002)

11. Xiao, L., Wei, Z., Ye, J.: Comments on robust embedding of visual watermarks using discrete wavelet transform and singular value decomposition and theoretical analysis. Journal of Electronic Imaging 17(4), 40501 (2008)

12. Zhang, T.X., Zheng, W.M., Lu, Z.M., Liu, B.B.: Comments on a semi-blind digital watermarking scheme based on singular value decomposition. In: Proceedings of International Conference on Intelligent Systems Design and Applications, November 2008, vol. 2, pp. 123-126 (2008) 
13. Loukhaoukha, K., Chouinard, J.Y.: On the security of ownership watermarking of digital images based on SVD decomposition. Journal of Electronic Imaging 19(1), 013007 (2010)

14. Cox, I., Kilian, J., Leighton, F.T., Shamoon, T.: Secure spread spectrum watermarking for multimedia. IEEE Transactions on Image Processing 6(12), 1673-1687 (1997)

15. Xianghong, T., Lu, L., Lianjie, Y., Yamei, N.: A digital watermarking scheme based on dwt and vector transform. In: Proceedings of International Symposium on Intelligent Multimedia, Video and Speech Processing, October 2004, pp. 635-638 (2004) 